\title{
La gestione integrata delle malerbe: un approccio sostenibile per il contenimento delle perdite di produzione e la salvaguardia dell'ambiente
}

\author{
Giuseppe Zanin, ${ }^{1}$ Aldo Ferrero, ${ }^{2}$ Maurizio Sattin ${ }^{3}$ \\ ${ }^{1}$ Dipartimento di Agronomia Ambientale e Produzioni Vegetali, Università di Padova; \\ ${ }^{2}$ Dipartimento di Agronomia, Selvicoltura e Gestione del Territorio, Università di Torino; \\ ${ }^{3}$ Istituto di Biologia Agroambientale e Forestale - CNR Legnaro, Italy
}

\begin{abstract}
The main issue that European agriculture is facing is related to maintaining and if possible increasing yields while reducing inputs especially pesticides, including herbicides. Society is paying increasing attention to the quality of products as well as to the sustainability of the productive system. Control of weeds is necessary to achieve economically acceptable yields. The main tool is chemical weed control. This practice spread quickly after its introduction, due its efficacy and simplicity, but it also generated a series of agronomic and environmental issues. One of the solutions to maintain yields, while reducing herbicide use is the adoption of the Integrated Weed Management (IWM). IWM is based on the knowledge of weeds and their response to cropping practices, weed-crop competitive relationships and chemical and non-chemical means of control. IWM has had a quite remarkable success among researchers, politicians and public opinion, but has rarely been applied by farmers. The limited implementation of this management approach may have different explanations: i) IWM is more complex than conventional management as it requires greater skill and is more time consuming; ii) there is a lack of financial sup-
\end{abstract}

Correspondence: Prof. Giuseppe Zanin, Dipartimento di Agronomia Ambientale e Produzioni Vegetali, Università di Padova, viale dell'Università 16, 35020 Legnaro (PD), Italy.

Tel. +39.049.8272819 - Fax +39.049.8272839.

E-mail: giuseppe.zanin@unipd.it

Key words: decision support systems, integrated management, predictive models, proactive agronomic practices, risk evaluation, weeds.

Parole chiave: gestione integrata, malerbe, modelli previsionali, pratiche agronomiche proattive, sistemi di aiuto alle decisioni, valutazione del rischio.

Received for publication: 20 April 2011.

Accepted for publication: 4 August 2011.

Società rappresentata nell'ambito AISSA: Società Italiana per la Ricerca sulla Flora Infestante - SIRFI

CCopyright G. Zanin et al., 2011

Under no circumstances figures can be used without prior written consent of the copyright owner.

Licensee PAGEPress, Italy

Italian Journal of Agronomy 2011; 6(s2):e6

doi:10.4081/ija.2011.6.s2.e6

This work is licensed under a Creative Commons Attribution NonCommercial 3.0 License (CC BY-NC 3.0). port and demonstration farms, necessary to convince the farmers to adopt it; iii) IWM is considered riskier than conventional management; iv) research has not succeeded in transferring scientific knowledge to the productive systems.The paper includes an analysis of several examples of IWM application in Italy in recent years (development of decision support systems, predictive models, formation of GIRE (Italian Herbicide Resistance Working Group) with the objective of defining their benefits and drawbacks and finding new solutions for the future. It is also underlined that the main difficulty in IWM development is the lack of integration between means of control and proactive agronomic practices that are suitable to prevent the spread and evolution of weeds in the fields.

\section{Riassunto}

L'attuale problema dell'agricoltura è quello di mantenere 0 meglio aumentare le rese riducendo l'impiego di mezzi tecnici in particolare gli agrofarmaci e tra questi gli erbicidi. La società civile è infatti sempre più attenta non solo alla qualità dei prodotti ma anche alla sostenibilità del sistema produttivo. Il controllo delle malerbe è necessario per garantire livelli di produzione economicamente accettabili. Il mezzo principale è il diserbo chimico che, da subito, si è imposto per la sua efficacia e semplicità ma ha creato anche vari problemi agronomici ed ambientali. La soluzione per mantenere le rese riducendo l'impiego di erbicidi è il ricorso all'Integrated Weed Management (IWM). L'impiego dell'IWM si basa sulla conoscenza delle malerbe e della loro risposta alle tecniche colturali, delle relazioni competitive tra malerbe e coltura e dei mezzi di controllo non solo chimici. L'IWM ha suscitato grande interesse presso i ricercatori, i politici e l'opinione pubblica ma ha avuto scarso seguito presso gli agricoltori. Diverse sono le ragioni del limitato sviluppo di questo approccio operativo: l'IWM è una gestione più complessa della tradizionale che richiede più conoscenze e più tempo, mancano incentivi economici per convincere gli agricoltori ad utilizzarlo e mancano le aziende dimostrative, l'IWM è ritenuto una gestione più rischiosa di quella tradizionale, la ricerca non è stata in grado di trasferire efficacemente le conoscenze di base nel processo produttivo. Viene poi eseguita una valutazione critica sugli esiti di alcuni tentativi di IWM sviluppati in Italia negli ultimi anni (Sistemi di Aiuto alle Decisioni, modelli previsionali, costituzione del GIRE, Gruppo Italiano Ricerca sulla Resistenza) nel tentativo di individuare i punti di debolezza e di forza di tali proposte applicative e trovare soluzioni nuove per il futuro. La difficoltà maggiore che viene evidenziata è la mancanza di integrazione tra mezzi di controllo e le pratiche agronomiche definite proattive in grado cioè di rendere il campo coltivato il meno favorevole possibile all'insediamento e sviluppo delle malerbe. 


\section{Definizione del problema}

L'attività agricola è oggi principalmente finalizzata ad ottenere produzioni sempre più elevate con tecniche di gestione meno dipendenti dai fattori di produzione non rinnovabili (concimi, agrofarmaci, carburanti ecc.) (Tilman et al., 2002).

Il raggiungimento di tali obiettivi in Italia è però contrastato da due importanti criticità: i) la continua perdita di terreno agricolo a causa dell'urbanizzazione che interessa purtroppo le aree agricole più produttive del Paese; ii) la salvaguardia ambientale sostenuta da crescenti vincoli legislativi a livello europeo e nazionale e invocata da un'opinione pubblica sempre più attenta non solo alla qualità dei prodotti ma anche alla sostenibilità del processo produttivo.

Particolare significato assume in questo quadro la pratica della gestione delle malerbe. Nonostante le contromisure messe in atto, la perdita dovuta all'azione competitiva delle malerbe a livello mondiale è pari al 13,2\% (Zoschke e Quadranti, 2002). Se non si mettessero in atto sistemi di controllo la perdita raggiungerebbe complessivamente il $34 \%$ (Oerke, 2006) a livello mondiale, stima simile a quelle di Zanin et al. (1992) e di Vidotto e Ferrero (2009) per l'Italia: 23-30\% nel grano, 34$37 \%$ nel mais, $33,3 \%$ nella soia, $50 \%$ nella bietola e superiore al $50 \%$ nel riso. La produzione agraria, almeno quella riguardante le specie annuali, non può quindi prescindere dal controllo delle malerbe.

A partire dagli anni ' 60 questa pratica è stata realizzata in modo efficace ed economico grazie allo sviluppo di diserbanti sempre più affidabili. L'impiego continuo, e spesso esclusivo, di tali prodotti ha però favorito nel tempo lo sviluppo di una serie di problemi agronomici (comparsa e sviluppo di flore di compensazione e di popolazioni resistenti) ed ambientali (contaminazione delle acque profonde e superficiali) che richiedono una tempestiva ed adeguata soluzione (Ruegg et $a l .$, 2007). La sfida è quindi quella di mettere a punto sistemi di gestione delle malerbe che siano economici, efficaci e con un ridotto impiego di erbicidi.

La soluzione ritenuta più efficace è l'applicazione della gestione integrata delle malerbe (Integrated Weed Management, IWM), sistema che Shaw (1982), ha così definito: la migliore combinazione di misure agronomiche, colturali, biologiche e chimiche che permette, nelle specifiche situazioni, di gestire le malerbe nella maniera più economica, più favorevole all'ambiente e socialmente accettabile.

L'IWM è un approccio di sistema basato principalmente sulla conoscenza della biologia delle malerbe e delle interazioni tra malerbe e colture e tra malerbe e tecniche colturali. Nell'IWM le conoscenze sostituiscono in parte i mezzi di lotta (Pretty, 2008).

L'IWM si basa su misure proattive e su misure reattive (Sullivan, 2003). Le misure proattive creano le condizioni perché il sistema colturale risulti sfavorevole all'insediamento, alla crescita ed alla competizione delle malerbe; le misure reattive sono gli interventi di controllo che vengono messi in atto dopo che i problemi sono sorti. Le misure proattive comprendono un insieme di accorgimenti agronomici, già conosciuti da tempo, le cosiddette pratiche agronomiche soppressive (weed-suppressive agronomic practices), che creano un ambiente ecologico sfavorevole all'insediamento delle malerbe e concorrono a mantenere bassa la loro densità a tal punto da rendere sufficienti misure reattive meno aggressive ed impattanti (es. erbicidi a dosi più basse, impiego di soli interventi meccanici) (Liebman et al., 2001; Anderson, 2007). Quasi tutti i segmenti della tecnica colturale possono svolgere un ruolo preventivo che viene esaltato dallo loro opportuna combinazione, come la gestione del terreno, la rotazione delle colture, dei residui colturali, dell'intercoltura, della fertilizzazione, l'impiego di colture di copertura. La conoscenza del funzionamento dell'agroecosistema e degli effetti dei diversi interventi agronomici sulla dinamica delle popolazioni di malerbe rappresenta la base per l'applicazione di efficaci misure proattive: anche per le malerbe si deve passare, in sostanza, dalla cura ad una gestione di medio lungo periodo (Ferron e Deguine, 2005).

Le misure reattive comprendono l'uso di erbicidi, gli interventi meccanici, fisici e manuali. Soprattutto su questa componente dell'IWM si è concentrata l'attenzione della ricerca, tanto che l'IWM è stato, almeno inizialmente, inteso come l'approccio per usare meno e meglio gli erbicidi e solo limitatamente come l'approccio che, attraverso una dettagliata conoscenza dell'agroecosistema, delle sue componenti ed interazioni, è in grado di ridurre l'insediamento e la crescita delle malerbe e quindi rendere il ricorso agli erbicidi meno necessario. Già alla metà degli anni '90 Morse e Buhler (1997) evidenziavano il dualismo tra IPM (Integrated Pest Management) tattica e IPM strategica affermando che quest'ultima è la soluzione ideale, mentre l'IPM tattica è l'espressione debole dell'ideale.

\section{Cosa serve all'Integrated Weed Management?}

L'IWM è una componente dell'IPM ed incorpora conoscenze di biologia delle malerbe e di ecofisiologia delle colture (Sanyal et al., 2008). Anche nell'era degli erbicidi i problemi creati dalle malerbe persistono e il miglioramento delle conoscenze sulla loro biologia è essenziale per sviluppare e mettere in essere approcci gestionali efficaci (Van Acker, 2008).

Per impostare l'IWM servono molte e differenziate conoscenze a livello di seme, di plantula, di pianta adulta; bisogna poi conoscere la capacità competitiva delle varie malerbe nelle diverse colture e la loro risposta al variare degli interventi agronomici. Dei semi bisogna conoscere la temperatura ed il potenziale idrico di base, indispensabili per modellizzarne l'emergenza, il tipo di dormienza che influenza la scalarità di emergenza e la longevità nel terreno. Quest'ultimo carattere poi è profondamente influenzato dalla modalità di gestione del terreno e dalla profondità di interramento, così come il tasso di predazione che è un aspetto ancora non molto studiato ma che potrebbe aprire interessanti possibilità di intervento (Barberi et al., 2010). Bhowmik (1997), sostiene, a questo riguardo, che lo sviluppo di approcci integrati in grado di ridurre la banca semi interferendo con la dormienza o le esigenze germinative dei semi potrebbe migliorare le strategie di gestione delle malerbe e dare un forte impulso all'IWM.

Per quanto riguarda le piante è importante saperle riconoscere allo stadio di plantula, avere un'idea della finestra di emergenza e della loro prolificità. È utile, altresì, conoscere la dinamica delle popolazioni di malerbe in relazione alle agrotecniche applicate, e in particolare all' avvicendamento colturale, alla concimazione, alla lavorazione del terreno, alla gestione dell'intercoltura e dei residui colturali e al diserbo. La domanda a cui bisogna saper rispondere, almeno a grandi linee, è la seguente Cosa ci dobbiamo aspettare se l'agrotecnica cambia?

In assenza di lavorazioni si determina, ad esempio, un forte cambiamento della composizione floristica, con un significativo aumento delle emicriptofite, delle biennali, delle camefite, delle nanofanerofite, delle specie a disseminazione ornitocora ed anemocora, delle graminacee e delle composite e in generale di tutte le specie indifferenti (Zanin et al., 1997) (Figura 1). Se si vuole adottare la tecnica della non lavorazione è opportuno conoscere queste risposte adattative della flora spontanea e prevedere l'adozione di adeguati programmi di gestione. La diffusione di certe specie in specifici sistemi colturali è infatti la risultante dell'effetto combinato delle caratteristiche morfologiche e dell'ecofisiologia dei semi, del sistema di riproduzione e di moltiplicazione vegetativa, della modalità di dispersione dei semi, dell'approfondimento dell'apparato radicale, oltreché della tolleranza agli erbicidi.

Particolare importanza assume a questo riguardo anche la conoscenza della capacità competitiva delle malerbe nelle diverse colture ed in particolare degli indici competitivi in funzione della densità (i ed a) e 
degli indici competitivi temporali in funzione della durata e del periodo di competizione (Durata della Competizione Tollerata, DCT e Periodo Richiesto di Assenza dalle Malerbe, PRAM). La conoscenza di questi due parametri temporali permette poi di determinare il Periodo Critico, vale a dire il periodo di tempo durante il quale le infestanti devono essere controllate per prevenire perdite produttive di un livello definito (di solito il 5\%, che corrisponde a grandi linee, almeno per le colture principali alla soglia della perdita di resa accettabile) (Sattin e Tei, 2001).

Queste conoscenze vanno poi integrate con quelle relative alla fisiologia della coltura e in particolare ai vari aspetti in grado di modulare l'interazione competitiva tra coltura e malerbe quali ad esempio il tasso di crescita, la capacità di coprire rapidamente il terreno, la risposta all'azoto all' irrigazione, all'investimento. Rendere le colture più competitive attraverso il miglioramento genetico o la tecnica colturale ha importantissimi risvolti proattivi sull'insediamento e presenza delle malerbe nell'agroecosistema.

Lo sfruttamento di specie o cultivar selezionate per una accresciuta capacità allelopatica può giocare un ruolo importante all'interno dell'IWM: queste colture possono infatti essere utilizzate oltre che come coltura principale nell'ambito dell'avvicendamento anche come colture di copertura, mulch e smother crops, colture da sovescio, allargando i contesti di impiego dell'allelopatia (Tesio e Ferrero, 2010; Tesio et al., 2010; Khanh et al., 2005). La competitività delle colture può essere modificata anche attraverso un insieme di scelte messe in atto alla semina e che di solito sono svincolate dalle finalità di gestione delle malerbe, quali ad esempio la scelta della cultivar, la data, la densità e la profondità di semina, la disposizione spaziale, la localizzazione e l'epoca di concimazione. Combinando opportunamente queste scelte, si persegue l'obiettivo di contenere l'utilizzazione da parte delle malerbe delle risorse ambientali limitanti (acqua, luce, elementi nutritivi) sfavorendone l'insediamento.

Molto ampie sono poi le conoscenze da avere per quanto riguarda i mezzi di controllo. Degli erbicidi bisogna conoscere l'efficacia (kill rate) in funzione dello stadio di sviluppo e delle condizioni pedo-climatiche, la dose di impiego, la possibilità di miscelazione, la persistenza (tempo di dimezzamento), il meccanismo di azione, la propensione a sviluppare resistenze (basata sulla classificazione HRAC), le caratteristiche chimico-fisiche e partitive che modulano il comportamento

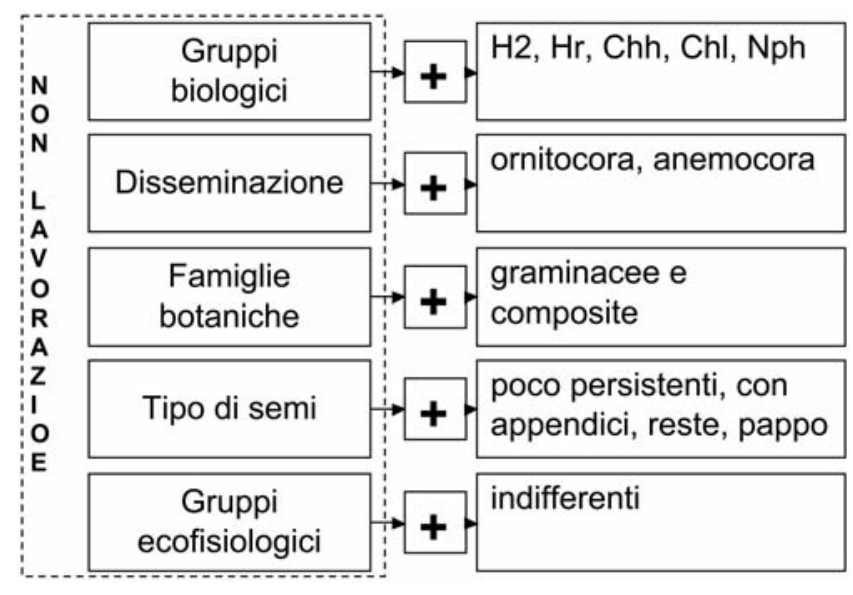

Figura 1. Risposta delle piante infestanti alla non lavorazione. $\mathrm{H} 2$, piante biennali; $\mathrm{Hr}$, piante emicriptofite; $\mathrm{Chh}$, piante camefite erbacee; Chl, piante camefite legnose; Nph, piante nanofanerofite.

Figure 1. Response of weed communities to no till. H2, biennial plants; $\mathrm{Hr}$, hemicryptophytes; Chh, herbaceous chamaephytes; Nph, dwarf phanerophytes. ambientale degli erbicidi.

L'IWM richiede un complesso di conoscenze molto vasto e soprattutto dinamico, legato alla continua variazione del panorama degli erbicidi introdotti 0 eliminati dal mercato e alla progressiva evoluzione del quadro floristico, per l'introduzione di nuove piante esotiche o preadattate (sensu Cousens e Mortimer, 1995). In queste condizioni, ogni 4-5 anni, si rende necessaria la rimodulazione del programma di gestione delle malerbe, con un continuo impegno di aggiornamento richiesto ai singoli agricoltori o messo a loro disposizione da un aggiornato servizio di assistenza tecnica.

\section{Perché l'Integrated Weed Management è poco utilizzato dagli agricoltori?}

L'IWM ha suscitato grande interesse presso i ricercatori, i politici e l'opinione pubblica ma ha avuto scarso seguito presso gli agricoltori. Perché la pratica dell'IWM è poco utilizzata? Perché gli agricoltori dovrebbero seguire protocolli creati dai ricercatori per i ricercatori? Alcune possibili commenti a tali domande sono i seguenti: i) l'IWM è una gestione più complessa della tradizionale, sia per le conoscenze richieste e sia per la necessità di integrare le stesse a livello tattico e strategico. Sono proprio le conoscenze biologiche ed agronomiche quelle che permettono di individuare gli strumenti in grado di surrogare il ricorso agli erbicidi. Del resto il grande successo di questi ultimi è principalmente motivato dalla possibilità di controllare le malerbe anche senza un'approfondita conoscenza della loro biologia e del sistema colturale. L'applicazione dell'IWM richiede da parte degli agricoltori un forte investimento nell'apprendimento (Pretty, 2008). ii) non vengono messi a disposizione incentivi economici per incoraggiare gli agricoltori ad utilizzarla e mancano le aziende dimostrative. La riforma Fischler del 2003 ha innovato la politica agricola europea, legando la concessione del sostegno al rispetto di una serie di obblighi rappresentativi di valori desiderabili dal punto di vista ambientale e sociale (De Castro, 2010); il sostegno è condizionato in sostanza dall'adozione di certi comportamenti attivi, e tra questi figura anche l'adozione dell'IWM, nei confronti della società. A questo riguardo è però il caso di chiedersi se l'entità di questi incentivi è tale da indurre gli agricoltori ad adottare l'IWM ed in particolare ad adottare le misure proattive. Con tutta probabilità solo un più elevato livello degli incentivi economici renderebbe più rapido il processo di transizione verso un'agricoltura sostenibile (Pretty, 2008). Un ruolo fondamentale nell'accettazione dei sistemi innovativi è rappresentato poi, anche, dalle aziende dimostrati$v e$. Queste sono il più efficace metodo di trasferimento tecnologico dell'innovazione nel settore agricolo e nella gestione delle malerbe in particolare (Sanyal et al., 2008). L'agricoltore infatti si deve convincere che una certa soluzione è preferibile ad un'altra e lo si convince non a parole ma facendogli vedere sul campo l'esito di una certa soluzione a confronto con altre. Il servizio di assistenza tecnica nel nostro Paese purtroppo è stato smantellato e ricostituirlo non è facile. Moss (2008) afferma che poche risorse sono state allocate alle iniziative di trasferimento tecnologico in UK, e ciò è ancora più vero per l'Italia. iii) L'IWM è considerato un sistema di gestione più rischioso di quello tradizionale (Greene et al., 1985; Swanton et al., 2008). Questo punto di vista in realtà non è unanimemente condiviso. Secondo Jones et al. (2006) in condizioni deterministiche, cioè ripetendo negli anni gli stessi interventi gestionali, l'IWM aumenta il beneficio economico del 6\% rispetto ai sistemi convenzionali di gestione delle malerbe. Questo valore è considerato troppo basso per indurre gli agricoltori ad adottare il sistema. In condizioni stocastiche, che considerano cioè la variabilità spaziale $\mathrm{e}$ stagionale e la conseguente differente risposta gestionale tattica, il vantaggio aumenta di dieci volte. Nel valutare tecnologie come l'IWM che coinvolgono risposte biologiche e dinamiche complesse, l'impatto 
della variabilità stagionale e la sequenza delle decisioni che devono essere prese non può essere ignorata. Anche Wu (2010) sostiene che la gestione basata su regole dinamiche che si adattano nel tempo alle condizioni climatiche e floristiche aumenta il margine di profitto per l'agricoltore senza richiedere un aumento delle quantità di erbicidi. I confronti tra sistemi diversi di controllo non dovrebbero mai essere eseguiti in condizioni statiche come purtroppo avviene nella maggior parte delle sperimentazioni. Da queste considerazioni nasce anche la necessità di sviluppare idonei metodi statistici in grado di stimare meglio i rischi legati all'applicazione dell'IWM; in tal senso la collaborazione tra agronomi-malerbologi ed economisti agrari sarebbe quanto mai auspicabile. iv) La ricerca non è stata in grado di trasferire efficacemente a livello operativo le conoscenze acquisite sul piano sperimentale. Probabilmente perché gli strumenti proposti sono troppo complicati o costosi o richiedono un livello di organizzazione non ancora presente. Il coinvolgimento degli utilizzatori finali del lavoro di ricerca nella definizione delle priorità e nella fase di trasferimento tecnologico è considerato fattore chiave per il successo del controllo integrato delle malerbe, e della difesa integrata più in generale. Il contributo della ricerca nel settore della biologia ed ecologia delle malerbe alla soluzione dei problemi malerbologici è, nella percezione degli stessi ricercatori di settore, modesto (Forcella, 1997; Bhowmik, 1997; Mortensen et al., 2000; Norris, 1997; Sanyal, 2008).

Perché questo tipo di ricerca ha un impatto percepito così basso? Perché non si è riusciti a tradurre le conoscenze in scelte gestionali facilmente inseribili nei diversi sistemi colturali e perché gli strumenti sviluppati (es. modelli di simulazione e Sistemi di Aiuto alle Decisioni, SAD) sono rimasti confinati nei laboratori di ricerca?

Secondo Moss (2008), i ricercatori spesso non hanno valutato le difficoltà e le problematiche del trasferimento dei risultati dalla scala di laboratorio, serra o parcella a quella di pieno campo, a livello aziendale. A ciò si aggiunge anche la mancanza di consapevolezza della complessità e delle risorse necessarie per operare il trasferimento dei risultati da una scala all'altra. Nell'applicazione a livello aziendale di GESTINF (Berti et al., 2003) si è osservato che le soluzioni che il modello proponeva erano personalizzate campo per campo e comportavano un numero di soluzioni chimiche nettamente superiore rispetto all'unica scelta dall'azienda con un aggravio dei costi di distribuzione. Impiegando GESTINF si doveva considerare un'ulteriore componente di costo oltre a quella dello scouting; questa necessità inizialmente non era stata messa a fuoco.

I modelli sono ritenuti da tutti un importante strumento operativo per l'applicazione dell'IWM; tuttavia gli studi sui modelli si sono prevalentemente concentrati su alcuni singoli aspetti (gestione della resistenza agli erbicidi, riduzione dell'input chimico, rapporti competitivi tra malerbe e colture) e solo pochi hanno riguardato la gestione delle malerbe a livello del complesso sistema aziendale, nel medio-lungo periodo. Pochi ricercatori hanno esplorato i rischi economici e sociali connessi all'impiego di certe soluzioni. Anche limitandosi a considerare i modelli sulle malerbe si può osservare come questi si siano concentrati principalmente sul controllo chimico e sulla individuazione della soglia di infestazione oltre la quale conviene trattare (Doyle, 1997). Così i mezzi non chimici hanno avuto poca attenzione da parte della ricerca, così come la loro integrazione con quelli chimici. Il criterio principalmente usato per definire le soluzioni migliori è stato quello dei costi; altri criteri, come la riduzione del danno ambientale, sono stati ignorati, forse anche per la difficoltà intrinseca di definirli. In sostanza il contributo dei modelli alla comprensione dei complessi meccanismi sottesi alla realizzazione dell'IWM è stato piuttosto limitato, compromettendo l'impatto della ricerca a livello pratico.

Merita riportare anche l'interessante considerazione di Neve et al. (2009), secondo cui la ridotta incidenza della ricerca di settore a livello pratico è dovuta anche al fatto che la malerbologia si è staccata dalla botanica e dalla ecologia vegetale, agganciandosi via via all'agronomia e alle produzioni vegetali. Questa associazione ha portato la malerbologia a focalizzarsi prioritariamente su fisiologia ed agronomia e a considerare le malerbe, alla stregua delle colture, una componente dotata di uniformità all'interno dell'agroecosistema, disconoscendo la loro peculiarità e cioè l'estrema variabilità e capacità di evolvere in risposta alle pressione degli interventi umani. La comprensione di queste dinamiche è la condizione essenziale per individuare e progettare soluzioni gestionali sostenibili.

\section{Valutazione critica di alcuni tentativi di applica- zione dell' l'Integrated Weed Management in Italia}

Anche nel nostro paese sono stati sviluppati alcuni strumenti e avviate delle esperienze per favorire l'impiego dell'IWM. Di seguito vengono presi in considerazione alcuni esempi significativi.

\section{Sistema di Aiuto alle Decisioni GESTINF}

GESTINF è un SAD che indica la/le soluzioni economicamente più convenienti sulla base di sopralluoghi (scouting) necessari per definire la composizione quanti-qualitativa della flora infestante presente in un certo appezzamento (Berti e Zanin, 1997). Si basa essenzialmente sul concetto di densità equivalente, attraverso il quale si riesce a prevedere la perdita di resa prodotta da una flora mista (Berti e Zanin, 1994). Il sistema indica la soluzione economicamente migliore e la densità di malerbe dopo gli interventi, questo elemento è utile in quanto dà un 'idea anche della disseminazione prodotta dall'infestazione residua, cioè del danno indiretto. Inoltre calcola un indice ambientale che indica l'impatto potenziale dei diversi erbicidi sulle acque di falda (Berti et al., 1995). Questo modello è stato validato sul frumento e sulla soia. E'stato utilizzato per due anni consecutivi in una grande azienda del litorale veneto (Rossi et al., 1997), in Piemonte (Ferrero et al., 1997), e per un paio di anni dai tecnici della Coldiretti della Provincia di Treviso. GESTINF è stato di fatto utilizzato unicamente nell'ambito della ricerca. È un sistema che ha richiesto un impegno finanziario di circa 600 milioni di lire (Zanin, 1997) che ha però avuto scarsa diffusione presso gli agricoltori; il suo sviluppo nel tempo ha prodotto un buon numero di lavori scientifici, ma questi lavori non hanno avuto ricadute di rilievo sul piano applicativo. Sui motivi per cui questo sistema di aiuto alle decisioni non ha avuto successo si possono avanzare alcune ipotesi.

Il sistema viene ritenuto dagli agricoltori troppo complicato, tanto che nei pochi casi di utilizzo in pieno campo è stato necessario il supporto di un tecnico. L'assistenza da parte di un tecnico era stata messa in conto, ma si riteneva che dovesse limitarsi ad uno o due anni per consentire all'agricoltore di avere un sufficiente grado di autonomia; in realtà terminata la fase di formazione l'agricoltore ha abbandonato il sistema.

Per un buon funzionamento il sistema richiede uno scouting accurato ed eseguito con criteri statistici corretti, per evitare l'utilizzazione di dati non rappresentativi e quindi l'ottenimento di risposte non soddisfacenti (Berti et al., 1992). In relazione a queste esigenze si rende necessario conoscere le malerbe allo stadio di plantula, ed effettuare frequenti sopralluoghi sui campi, dedicando tempo per una azione che rappresenta di per sé la parte qualificante ed innovativa del processo decisionale. Gli agricoltori hanno affidato ai tecnici l'operazione di scouting, ritenendola una perdita di tempo, un'azione curiosa, ed in ogni caso costosa. Il costo in effetti è un fattore chiave; in una situazione in cui i margini di redditività si restringono sempre più l'aggiunta di una nuova voce di costi al processo produttivo non è certamente ben accettata. Va ancora considerato che GESTINF non tiene conto del danno indiretto delle malerbe dovuto alla disseminazione, elemento 
particolarmente importante per l'agricoltore.

Il tempo intercorso tra la messa a punto di questo SAD e il suo utilizzo è risultato forzatamente lungo e quindi quando é risultato pronto per l'impiego si era già registrata una variazione nel quadro degli erbicidi disponibili sul mercato e delle malerbe presenti nelle colture agrarie. I data base del sistema richiedono un costante aggiornamento, non facilmente realizzabile in mancanza di specifiche risorse finanziarie. Questo è un problema particolarmente sentito in Italia dove mancano gli organismi di collegamento tra la ricerca e gli utilizzatori finali, in grado di cogliere l'innovazione, mantenerla aggiornata e proporla agli agricoltori.

$\mathrm{Al}$ riguardo, va altresì sottolineato che agli agricoltori servono non solo indicazioni sulle soluzioni ottimali dal punto di vista economico ma anche informazioni su altri aspetti decisionali quali ad esempio l'epoca più favorevole di intervento. Come si vede dalla Figura 2 il processo decisionale è complesso e, nella sua configurazione originaria, GESTINF è in grado di fornire solo alcune delle risposte richieste (se e come diserbare).

\section{II modello Alertinf}

Un altro esempio di sistema di aiuto alle decisioni è rappresentato da Alertinf, uno strumento messo a punto per prevedere l'emergenza delle malerbe nel mais (Masin et al., 2010). Questo sistema è stato inserito nel sito dell'ARPA del Veneto ed è utilizzabile liberamente dagli agricoltori; basta cliccare la stazione meteo ARPA più vicina all'azienda del richiedente, l'icona con l'infestante ed inserire la data di semina per ottenere in tempo reale la percentuale di emergenze raggiunta dalla stessa infestante. Nel 2010 primo anno di prova il sito ha avuto quasi 2000 visite.

Prevedere le emergenze è la base per razionalizzare i programmi di lotta alle malerbe sia per quanto riguarda l'impiego del mezzo chimico, sia per la realizzazione degli interventi meccanici in particolare quelli basati sulla falsa semina. Sono in corso altri studi volti a mettere a

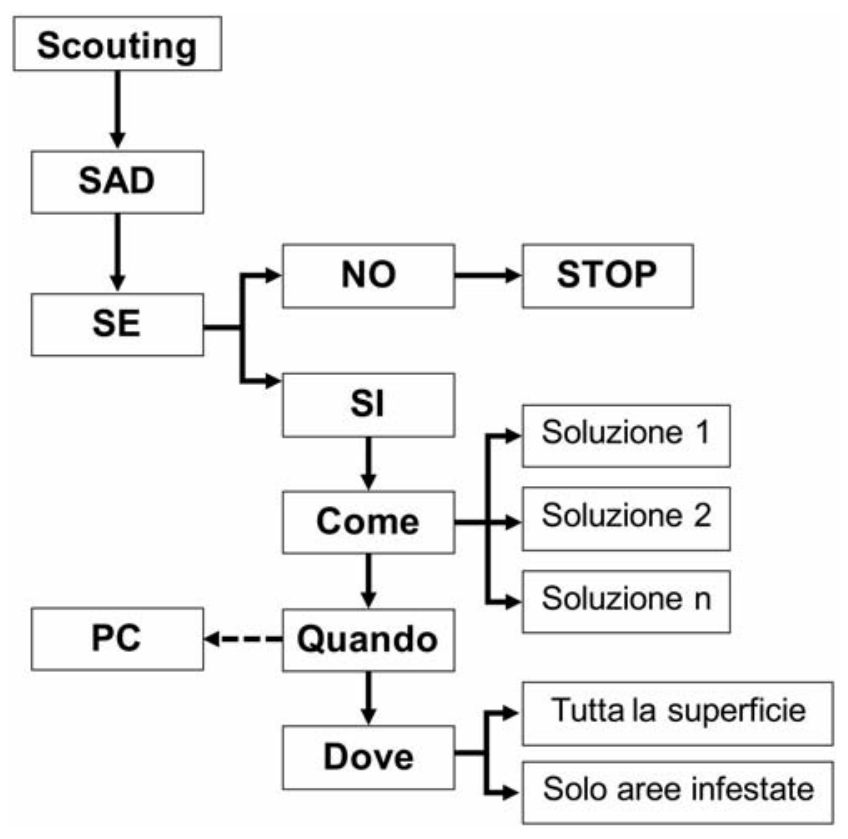

Figura 2. Scelte decisionali che l'agricoltore deve prendere nei trattamenti di post-emergenza. SAD, Sistema di Aiuto alle Decisioni (es. GESTINF); PC, periodo critico.

Figure 2. Decisional choices that farmers must make for postemergence treatments. SAD, Decision Support System (i.e GESTINF); PC, critical period. punto un sistema più completo dei precedenti, basato sull'integrazione di Alertinf e GESTINF per fornire informazioni più complete non solo di carattere qualitativo, ma anche quantitativo (Masin et al., 2011). E' comunque sempre da chiedersi se sono proprio questi gli strumenti che gli agricoltori si aspettano ed in ogni caso bisogna sottolineare che questi strumenti si inseriscono principalmente nella IPM tattica.

\section{Gruppo Italiano di lavoro sulla Resistenza agli Erbicidi: esempio di azione integrata e condivisa}

Un'iniziativa di successo nei rapporti tra i vari attori del sistema è rappresentato dal GIRE (Gruppo Italiano di lavoro sulla Resistenza agli Erbicidi; www.resistenzaerbicidi.it), un gruppo formato da rappresentanti della ricerca pubblica, dell'università, dell'assistenza tecnica e delle società produttrici di agrofarmaci, interessate a prevenire e contenere il fenomeno della resistenza agli erbicidi in Italia. La missione del GIRE, in accordo con quella dell'HRAC (Herbicide Resistance Action Committee) e del gruppo di lavoro dell'EWRS (European Weed Research Society) sulla resistenza agli erbicidi, è principalmente quella di:

facilitare un'efficace gestione della resistenza, favorendo la cooperazione e la comunicazione tra organismi pubblici sia di ricerca che accademici, le industrie e gli operatori del settore, allo scopo di promuovere un utilizzo responsabile degli erbicidi;

promuovere e contribuire ad una migliore comprensione delle cause e delle conseguenze della resistenza;

promuovere le strategie di gestione della resistenza basate sulla gestione integrata delle malerbe attraverso la pubblicazione di lineeguida pratiche;

supportare e partecipare a ricerche, conferenze e seminari che contribuiscano a migliorare le conoscenze sulla resistenza agli erbicidi; stimolare la collaborazione tra ricercatori pubblici e privati.

È un gruppo conosciuto, che ha acquisito autorevolezza e credibilità. Uno dei messaggi di base del GIRE, in accordo con Murphy e Lemerle (2006), è che gli agricoltori devono essere proattivi nel proteggere la longevità degli erbicidi disponibili, riducendo la probabilità di selezionare popolazioni resistenti attraverso l'implementazione dell'IWM. Gli interessi, convergenti in questo caso tra agricoltori ed industria, fanno ben sperare che almeno in questo ambito gli agricoltori possano essere spinti a scegliere sistemi gestionali virtuosi. Wilson et al. (2008) sottolineano che salvaguardare l'efficacia degli erbicidi prevenendo l'insorgenza della resistenza è uno dei principali obiettivi dell'IWM. Llewellyn et al. (2007) evidenziano in questo ambito la necessità di una approfondita valutazione economica della gestione della resistenza, soprattutto per quanto riguarda i costi delle misure di prevenzione.

\section{Conclusioni}

Non deve sembrare eccessivo affermare che le azioni da mettere in campo si conoscono. Cuppari (1896), allievo di Ridolfi, in Lezioni di Agricoltura tracciava già le linee per una impostazione proattiva della gestione delle malerbe. Ecco alcuni brevi passi:

Le cattive erbe poi invadono tanto più le dimestiche quanto meno le trovano adatte ai terreni che occupano e quanto più frequentemente riseminate.

In natura le piante di diversa indole sono consociate, vivono le une accanto alle altre, e si cedono a vicenda il posto, dove si alternano. L'arte contrariando questa legge naturale vuole ricoprire larghe falde di terreno colla stessa pianta, che vi fa tornare di frequente. Non è quindi da fare meraviglie, se le tendenze naturali tentano di riprendere il loro corso a dispetto dell'uomo, il quale è forzato a contenerle con cure eccessive. Da questo un avvicendamento ben concertato, allungando il giro e variando le colture, può far sostenere la lotta con meno spesa. 
L'opera del Cuppari è un trattato sull'IWM: a quel tempo del resto il controllo delle malerbe si basava quasi esclusivamente sulle misure proattive. L'avvento degli erbicidi, strumenti fondamentali nella gestione delle malerbe, ma responsabili talvolta anche di una eccessiva semplificazione delle pratiche colturali, ha portato a trascurare tutto questo bagaglio di conoscenze (Zanin e Catizone, 2003); non sorprende quindi che la componente reattiva dell'IWM sia quella che negli ultimi decenni ha avuto i maggiori approfondimenti in particolare sul versante dell'uso razionale e mirato degli erbicidi. In questo ambito l'applicazione delle tecnologie proprie dell'agricoltura di precisione (GPS e GIS) e della robotica può essere interessante al fine di ridurre l'impatto ambientale ed i costi del diserbo (Sakai e Upadhyaya, 2007; Slaughter et al., 2008). Queste innovazioni permetterebbero anche di ridurre 0 eliminare i costi dello scouting sul terreno (Swinton, 2005) che, come si è visto, è stato uno degli elementi che hanno maggiormente frenato gli agricoltori nell'utilizzo di strumenti quali ad esempio GESTINF. Come evidenzia Lopez-Granados (2010), le problematiche di tipo economico, tecnologico e procedurale (per esempio la scelta tra il remote weed detection oppure il proximal (on ground) weed detection, tra site specific weed management oppure variable rate technology for weed management) sono numerose e c'è ancora molto lavoro interdisciplinare da fare.

Molto meno si è fatto a livello di approccio proattivo, non tanto perché non ci siano le conoscenze, ma perché le stesse non sono state integrate all'interno dei diversi sistemi colturali. In sostanza il ricorso alle misure proattive è sporadico, settoriale e incompleto e questo penalizza tutto il sistema colturale. Bisogna confrontare, all'interno di sperimentazioni di lunga durata, differenti itinerari tecnici combinati con diverse soluzioni per il controllo delle malerbe. Si deve in sostanza partire dal sistema colturale che deve essere progettato sulla base delle misure proattive (es. avvicendamento delle colture, alternanza dei disturbi, continua 0 prolungata copertura del terreno da parte della vegetazione erbacea, rapida copertura del terreno da parte della coltura, ecc.). All'interno dello specifico sistema si adatteranno le misure reattive, considerando la variabilità interannuale. Un primo tentativo è stato fatto da Chikowo et al., 2009, testando per un lungo periodo diverse combinazioni di IWM all'interno di differenti sistemi colturali, ottenendo buoni risultati. L'IWM deve in sostanza essere integrato nell'IPM e questo nell'IFM (Integrated Farming Management) (Ferron e Deguine, 2005).

È mancata anche l'interazione tra agronomi e malerbologi a livello di modellizzazione dei sistemi colturali: i più importanti sistemi di simulazione (CROPSYST, DSSAT ecc.) non sono in grado di valutare l'interferenza delle malerbe, componente che può ridurre anche di oltre il $30 \%$ la resa della coltura, ponendosi così come una componente non trascurabile dell'agroecosistema.

Queste considerazioni evidenziano la fondamentale necessità di far collimare le priorità dell'agricoltore con quelle della ricerca e sperimentazione. A questo scopo c'è bisogno di capire meglio i modelli mentali che stanno alla base delle scelte degli agricoltori. Analisi di questo tipo sono state ad esempio realizzate da Wilson et al. (2008) per identificare i processi razionali e motivazionali utilizzati dagli agricoltori, ad esempio per scegliere tra agricoltura biologia e agricoltura tradizionale, tra lotta tradizionale chimica e IWM, tra OGM e non OGM. Questi elementi sono indispensabili per rendere la comunicazione e l'educazione più efficaci e quindi più incisivo a livello pratico il ruolo della ricerca di settore. In futuro la ricerca dovrà incorporare la dimensione umana (ad es. attitudine al rischio, diversa percezione di fronte a diversi problemi) nello studio e sviluppo di soluzioni utili per l'IWM (Riemens et al., 2010). Anche Doohan et al. (2010) hanno sostenuto l'importanza della dimensione umana nella gestione delle malerbe ponendosi alcune domande complesse a cui non è facile dare una risposta immediata: Perché gli agricoltori non adottano misure per ritardare lo sviluppo della resistenza? Perché intervengono contro le nuove malerbe quando proprio non ne possono fare a meno? Perché sono poco propensi a mettere in atto misure preventive? Perché sono restii ad adottare l'IWM?

I malerbologi hanno finora pensato che questi aspetti non fossero di competenza. In mancanza di una loro specifica attenzione altri studiosi si sono occupati del problema e questi argomenti trovano spazio sempre maggiore in riviste estranee al circuito agronomico come Sociologia Ruralis, Rural Sociology, Ecological Economics, Risks analysis in cui trovano collocazione lavori di sociologi ed economisti. Anche Lichtfouse (2010) enfatizza il ruolo e l'importanza della sociologia nello studio dei sistemi colturali, ritenendola una componente molto utile per allineare meglio il punto di vista dell'agricoltore con quello del ricercatore.

Va rilevato al riguardo che queste osservazioni erano già state avanzate da Radosevich e Ghersa (1990) con la descrizione delle relazioni tra le sei discipline scientifiche fondamentali per la malerbologia e cioè fisiologia e botanica, chimica, genetica, ecologia, economia e appunto sociologia. Nel procedere del tempo si è un po' perso di vista il ruolo di questa componente nell'aiutare i ricercatori a meglio finalizzare le proprie ricerche in modo da fornire risposte alle vere esigenze degli agricoltori. Questo disinteresse ha accentuato la divaricazione tra gli obiettivi dei ricercatori ed i bisogni degli agricoltori: in sostanza i ricercatori si sono impegnati poco per comprendere la logica e le necessità dell'agricoltore e le loro strade si sono allontanate. Secondo Moss (2008) la ricerca in malerbologia ha seguito un orientamento accademico votato essenzialmente al raggiungimento degli indici bibliometrici, indispensabili per la progressione di carriera dei ricercatori. Ciò è comprensibile in quanto la sperimentazione sull'IWM, essendo di più lunga durata e costosa, porta ad un numero minore di pubblicazioni ISI e quindi è meno appetita dai ricercatori. Tuttavia sono proprio queste le sperimentazioni più utili per gli agricoltori e indispensabili per dare le risposte che la società chiede.

Merita anche ricordare l'osservazione di Oerke (2006) secondo cui la percentuale di perdita di produzione a livello mondiale, nonostante l'intenso impiego di erbicidi, non è molto cambiata dagli anni '60 ad oggi; il continuo impegno dedicato dai diversi attori del sistema produttivo agricolo (industria degli erbicidi, miglioratori genetici, malerbologi e agricoltori) nella weed management arm race ha portato fino ad oggi ad un risultato di parità. Si può ipotizzare che questo risultato possa continuare ad essere ottenuto solo in presenza di una costante immissione nel mercato di nuove molecole con nuovi meccanismi di azione; se questa immissione, come si ha ragione di credere stia succedendo, dovesse rallentare allora sarebbe difficile mantenere anche l'attuale pareggio. Si è innescata dall'introduzione del diserbo chimico una classica spirale perversa: l'evoluzione di popolazioni di malerbe sempre più adattate ai nuovi strumenti di lotta ha favorito lo sviluppo di sempre più sofisticati sistemi di controllo, che hanno esercitato una ancora più forte pressione di selezione per l'adattamento delle malerbe. Più le tecnologie diventano avanzate più la selezione per la weediness si intensifica (Neve et al., 2009). Ciò evidenzia che anche, e forse soprattutto all'interno dell'agricoltura più innovativa, c'è bisogno di una visione integrata del sistema colturale e quindi di IWM. La decisione della Monsanto di dare agli agricoltori americani 20 dollari ad acro perché usino un erbicida di pre-emergenza in funzione antiresistenza per rallentare la diffusione di malerbe resistenti al glifosate nel cotone (Volkmann, 2010), pone in evidenza che anche l'agricoltura biotecnologica è vulnerabile e non può prescindere da una corretta gestione integrata delle infestanti.

L'aumento della popolazione mondiale, l'impossibilità di aumentare la superficie coltivabile, il crescente interesse per diete sempre più sofisticate, la destinazione di terreni alla coltivazione di colture da energia, le conseguenze negative del riscaldamento globale renderanno sempre più complessa e problematica la produzione di derrate alimentari. Si tratta di nuove ed importanti sfide per l'agricoltura, che 
richiederanno un sempre maggiore coordinamento tra il settore della ricerca e quello della produzione: in questo contesto crescerà la richiesta di programmi di IWM compatibili con gli obiettivi produttivi e di pratica applicazione.

\section{Bibiliografia}

Anderson R.L., 2007. Managing weeds with a dualistic approach of prevention and control. A review. Agron. Sustain. Dev. 27:13-18.

Barberi P., Burgio G., Dinelli G., Moonen A.C., Otto S., Vazzana C., Zanin G., 2010. Functional biodiversity in the agricultural landscape: relationships between weeds and arthropod fauna. Weed Res. 50:388-401.

Berti A., Bravin F., Zanin G., 2003. Application of decision-support software for postemergence weed control. Weed Sci. 51:618-627.

Berti A., Zanin G., 1994. Density Equivalent. A method for forecasting yield loss caused by mixed weed populations. Weed Res. 34:327332.

Berti A., Zanin G., 1997. GESTINF: A decision model for post-emergence weed management in soybean (Glycine max (L) Merr). Crop Prot. 16:109-116.

Berti A., Zanin G., Baldoni G., Grignani C., Mazzoncini M., Montemurro P., Tei F., Vazzana C., Viggiani P., 1992. Frequency-distribution of weed counts and applicability of a sequential sampling method to integrated weed management. Weed Res. 32:39-44.

Berti A., Zanin G., Otto, S., Trevisan M., Capri E., 1995. Evaluation of the cost-risk relationship of groundwater contamination in weed control of soybean. Eur. J. Agron. 4:491-498.

Bhowmik P.C., 1997. Weed biology: importance to weed management. Weed Sci. 45:349-356.

Chikowo R., Faloya V., Petit S., Munier-Jolain N.M., 2009. Integrated weed mangement systems allow reduced reliance on herbicides and long-term weed control. Agr. Ecosyst. Environ. 132:237-242.

Cousens R., Mortimer M., 1995. Dynamics of Weed Populations. Cambridge University Press, Cambridge, UK.

Cuppari P., 1896. Lezioni di Agricoltura. Edizioni Nistri, Pisa, Italy.

De Castro P., 2010. L'agricoltura europea e le nuove sfide. Donzelli Ed., Roma, Italy.

Doohan D., Wilson R., Canales E., Parker J., 2010. Investigating the human dimension of weed management: new tools of the trade. Weed Sci. 58: 03-510.

Doyle C.J., 1997. A review of the use of models of weed control in Integrated Crop Protection. Agr. Ecosyst. Environ. 64:165-172.

Ferrero A., Vidotto F., Saglia A.A., 1997. Esperienze di utilizzo di GESTINF in Piemonte. In: G. Zanin e I. Sartorato (eds.) Strumenti informatici per il controllo delle infestanti: dalla teoria alla pratica. Tipografia Gotica, Padova, Italy, pp 51-60.

Ferron P., Deguine JP., 2005. Crop protection, biological control, habitat management and integrated farming. A review. Agron. Sustain. Dev. 25:17-24.

Forcella F., 1997. My view. Weed Sci. 45:327.

Greene C.R., Kramer A., Norton G.W., Rajotte E.G., McPherson R.M., 1985. An economic anlaysis of soyabean integrated pest management. Am. J. Agric. Econ. 63:567-572.

Jones R., Cacho 0., Sinden J., 2006. The importance of seasonal variability and tactical responses to risk on estimating the economic benefits of integrated weed management. Agr. Econ. 35:245-256.

Khanh T.D., Chung M.I., Xuan T.D., Tawata S., 2005. The exploitation of crop allelopathy in sustainable agricultural production. J. Agron. Crop Sci. 191:172-184.

Lichtfouse E., Hamelin M., Navarrete M., Debaeke P., Henri A., 2010. Emerging agroscience. Agron. Sustain. Dev. 30:1-10.
Liebman M., Mohler C.I., Staver C.P., 2001. Ecological Management of Agricultural Weeds. Cambridge University Press, Cambridge, UK.

Llewellyn R.S., Lindner R.K., Pannell D.J., Powles S.B., 2007. Herbicide resistance and the adoption of integrated weed management by Western Australian grain growers. Agr. Econ. 36:123-130.

Lopez-Granados F. 2010. Weed detection for site-specific weed management: mapping and real-time approaches. Weed Res. 51:1-11.

Masin R, Cacciatori G., Zuin M.C., Zanin G. 2010. AlertInf: emergence predictive model for weed control in maize in Veneto. Rivista Italiana di Agrometeorologia 1:5-9.

Masin R., Vasileiadis V., Loddo D., Otto S., Zanin G., 2011. A single time survey method to predict the daily weed density for weed control decision-making. Weed Sci. 59:270-275.

Morse S., Buhler W., 1997. IPM in developing countries: the danger of an ideal. Integrated Pest Management Reviews, 2: 175-185.

Mortensen D.A., Bastiaans L., Sattin M., 2000. The role of ecology in the development of weed management systems: an outlook. Weed Res. 40:49-62.

Moss S.R., 2008. Weed research: is it delivering what it should? Weed Res. 48:389-393.

Murphy C.E., Lemerle D., 2006. Continuous cropping systems and weed selection. Euphytica 148:61-73.

Neve P., Vila-Aiub M., Roux F., 2009. Evolutionary-thinking in agricultural weed management. New Phytol. 184:783-793.

Norris R.F., 1997. Weed Science Society of America weed biology survey. Weed Sci. 45:343-348.

Oerke E.C., 2006. Crop losses to pests. J. Agr. Sci. 144:31-43.

Pretty J., 2008. Agricultural sustainability: concepts, principles and evidence. Phil. T. R. Soc. B 363:447-465.

Radosevich S.R., Ghersa C.M., 1990. Weed science: a microcosm of agriculture neckriddle. Proc. North-Central Weed Sci. Soc. 45:4-8.

Riemens M.M., Groeneveld R.M.W., Kropff M.J.J., Lotz L.A.P., Renes R.J., Sukkel W., van der Weide R.J., 2010. Linking farmer weed management behaviour with weed pressure: more than just technology. Weed Sci. 58:490-496.

Rossi E., Miotto P., Montanari R., 1997. Esperienze aziendali di utilizzo di GESTINF. In: G. Zanin e I. Sartorato (eds.) Strumenti informatici per il controllo delle infestanti: dalla teoria alla pratica. Tipografia Gotica, Padova, Italy, pp 41-50.

Ruegg W.T., Quadranti M., Zoschke A., 2007. Herbicide research and development: challenges and opportunities. Weed Res. 47:271-275.

Sakai K., Upadhyaya S., 2007. Advanced engineering approach to weed management. Weed Biol. Manag. 7:1-2.

Sanyal D., Bowmik P.C., Anderson R.L., Shrestha A., 2008. Revisiting the perspective and progress of integrated weed management. Weed Sci. 56:161-167.

Sattin M., Tei F., 2001. Malerbe componente dannosa degli agroecosistemi. In: P. Catizone e G. Zanin (eds.) Malerbologia. Patron Ed., Bologna, Italy, pp 171-245.

Shaw W.C., 1982. Integrated weed management systems technology for pest management. Weed Sci. 30(suppl.1):1-12.

Slaughter D.C., Giles D.K., Downey D., 2008. Autonomous robotic weed control systems: a review. Comput. Electron. Agr. 61:63-78.

Sullivan P., 2003. Principles of sustainable weed management for croplands. Available from: https://attra.ncat.org/attrapub/summaries/summary.php?pub=109

Swanton C.J., Mahoney K.J., Chandler K., Gulden R.H., 2008. Integrated Weed Management: knowledge-based weed management systems. Weed Sci. 56:168-172.

Swinton S.M., 2005. Economics of site-specific weed management. Weed Sci. 53:259-263.

Tesio F., Ferrero A., 2010. Allelopathy, a chance for sustainable weed management. Int. J. Sust. Dev. World 17:377-389.

Tesio F., Weston L.A., Vidotto F., Ferrero A., 2010. Potential allelopathic 
effects of Jerusalem artichoke (Helianthus tuberosus) leaf tissues. Weed Technol. 24:378-385.

Tilman D., Cassman K.G., Matsons P.A., Naylor R., Polasky S., 2002. Agricultural sustainability and intensive production practices. Nature 418:671-677.

Van Acker R.C., 2008. Weed biology serves practical weed management. Weed Res. 49:1-5.

Vidotto F., Ferrero A., 2009. Interactions between weedy rice and cultivated rice in Italy. Ital. J. Agron. 4:127-136.

Volkmann K., 2010. Monsanto offers millions in rebates to farmers. St Louis Business Journal. Available from: http://www.bizjournals.com/stlouis/news/2010/10/19/monsantooffers-millions-of-dollars-in.html

Zanin G., 1997. Presentazione dell'incontro. In G. Zanin e I. Sartorato (eds.) Strumenti informatici per il controllo delle infestanti: dalla teoria alla pratica. Tipografia Gotica, Padova, Italy, pp 11-14.
Zanin G., Berti A., Giannini M., 1992. Economics of herbicide use on arable crops in North-Central Italy. Crop Prot. 11:174-180.

Zanin G., Catizone P., 2003. Malerbologia: dalla metà del XIX a quella del XX secolo. In: I Georgofili, Quaderni 2003-I. Evoluzione dei mezzi di difesa fitosanitaria. Società Editrice Fiorentina, Firenze, Italy, 1:195-269.

Zanin G., Otto S., Riello L., Borin M., 1997. Ecological interpretation of weed flora dynamics under different tillage systems. Agr. Ecosyst. Environ. 66:177-188.

Zoschke A., Quadranti M., 2002. Integrated weed management-quo vadis? Weed Biol. Manag. 2:1-10.

Wilson R.S., Tucker M.A., Hooker N.H., LeJeune J.T., Doohan D., 2008. Perceptions and beliefs about weed management: perspectives of Ohio grain and produce farmers. Weed Technol. 22:339-350.

Wu J.J., 2001. Optimal weed control under static and dynamic decision rules. Agr. Econ. 25:19-130. 